\title{
Traumatic Brain Injury in Later Life Increases Risk for Parkinson Disease
}

\author{
Raquel C. Gardner, MD, 1,2 James F. Burke, MD, ${ }^{3}$ Jasmine Nettiksimmons, $\mathrm{PhD},{ }^{2,4}$ \\ Sam Goldman, MD, MPH, ${ }^{1,2}$ Caroline M. Tanner, MD, ${ }^{1,2}$ and \\ Kristine Yaffe, $\mathrm{MD}^{1,2,4,5}$
}

Objective: Traumatic brain injury (TBI) is thought to be a risk factor for Parkinson disease (PD), but results are conflicting. Many studies do not account for confounding or reverse causation. We sought to address these concerns by quantifying risk of PD after TBI compared to non-TBI trauma (NTT; defined as fractures).

Methods: Using inpatient/emergency department (ED) International Classification of Disease, Ninth Revision code data for California hospitals from 2005-2006, we identified patients aged $\geq 55$ years with TBI $(n=52,393)$ or NTT $(n=113,406)$ and without baseline PD or dementia who survived hospitalization. Using Kaplan-Meier estimates and Cox proportional hazards models (adjusted for age, sex, race/ethnicity, income, comorbidities, health care use, and trauma severity), we estimated risk of PD after TBI during follow-up ending in 2011 . We also assessed interaction with mechanism of injury (fall vs nonfall) and effect of TBI severity (mild vs moderate/severe) and TBI frequency (1 $\mathrm{TBI}$ vs $>1 \mathrm{TBI}$.

Results: TBI patients were significantly more likely to be diagnosed with PD compared to NTT patients (1.7\% vs $1.1 \%, p<0.001$, adjusted hazard ratio $[H R]=1.44,95 \%$ confidence interval $[\mathrm{Cl}]=1.31-1.58)$. Risk of PD was similar for TBI sustained via falls versus nonfalls (interaction $p=0.6$ ). Assessment by $\mathrm{TBI}$ severity (mild $\mathrm{TBI}: \mathrm{HR}=1.24,95 \%$ $\mathrm{Cl}=1.04-1.48$; moderate/severe $\mathrm{TBI}: \mathrm{HR}=1.50,95 \% \mathrm{Cl}=1.35-1.66)$ and $\mathrm{TBI}$ frequency $(1 \mathrm{TBI}: \mathrm{HR}=1.45,95 \%$ $\mathrm{Cl}=1.30-1.60 ;>1 \mathrm{TBI}: \mathrm{HR}=1.87,95 \% \mathrm{Cl}=1.58-2.21)$ revealed a dose response.

Interpretation: Among patients aged $\geq 55$ years presenting to inpatient/ED settings with trauma, TBI is associated with a $44 \%$ increased risk of developing PD over 5 to 7 years that is unlikely to be due to confounding or reverse causation.

ANN NEUROL 2015;77:987-995

ncidence of traumatic brain injury (TBI) peaks 3 times over the lifespan: in childhood, in adolescence, and in older adulthood. ${ }^{1}$ Some prior studies have implicated any lifetime history of TBI as a risk factor for Parkinson disease (PD) ${ }^{2-4}$ the second most common neurodegenerative disease of aging. Other studies, however, have found no such association and raise the hypothesis that recall bias or reverse causation may contribute to the positive reported associations. ${ }^{5,6}$ Whether TBI sustained in older adulthood increases short-term risk of PD is a question that has proven particularly difficult to approach. Specifically, when evaluating risk of PD following TBI sustained in older adulthood-at a time when the cause of injury is overwhelmingly due to falls ${ }^{7,8}$ - it becomes increasingly likely that the patient fell and sustained the TBI due to early motor symptoms of $\mathrm{PD}$ rather than the reverse.

In this study, we sought to quantify risk of PD after recent TBI sustained in older adulthood. To mitigate potential confounding and reverse causation, we compared patients with TBI to those with other types of non-TBI trauma (NTT; eg, fracture). Even among patients who sustain TBI or NTT due to falls, however, it is conceivable that those who fall and sustain a head injury are more likely to have incipient PD due to slower reaction times and reduced ability to redirect the fall trajectory or break the fall with their a'rms. ${ }^{9}$ Thus, to

View this article online at wileyonlinelibrary.com. DOI: 10.1002/ana.24396

Received Jan 8, 2015, and in revised form Feb 16, 2015. Accepted for publication Feb 22, 2015.

Address correspondence to Dr Gardner, 4150 Clement St, Neurology \#127, San Francisco, CA 94121. E-mail: raquel.gardner@ucsf.edu

From the ${ }^{1}$ Department of Neurology, University of California, San Francisco, San Francisco, CA; ${ }^{2}$ San Francisco Veterans Affairs Medical Center, San Francisco, CA; ${ }^{3}$ Department of Neurology, University of Michigan and Department of Veterans Affairs, VA Center for Clinical Management and Research, Ann Arbor VA Healthcare System, Ann Arbor, Ml; ${ }^{4}$ Department of Epidemiology and Biostatistics, University of California, San Francisco, San Francisco, CA; and ${ }^{5}$ Department of Psychiatry, University of California, San Francisco, San Francisco, CA 
further mitigate potential reverse causation, we assessed for interactions with mechanism of injury. To further enhance causal inference, we assessed the role of age, TBI severity, TBI frequency, and time lag from trauma to PD diagnosis. We hypothesized that younger patients may be more resilient to the effects of mild TBI. ${ }^{8}$ We hypothesized that TBI would increase risk for PD in a dosedependent manner (greater for severe TBI compared to mild TBI; greater for multiple TBIs compared to single TBI). Furthermore, we hypothesized that although the estimated risk might be attenuated by excluding patients with falls or PD diagnoses soon after trauma (as these populations may be enriched for incipient PD), the effect would persist, thereby supporting a causal association between TBI and PD.

\section{Patients and Methods \\ Design}

This is a retrospective cohort study of administrative health data using the State Inpatient Databases (SID) ${ }^{10}$ and State Emergency Department Databases (SEDD) $)^{11}$ for the state of California, managed by the Healthcare Cost and Utilization Project (HCUP) and Agency for Healthcare Research and Quality. The SID and SEDD capture all inpatient and emergency department (ED) discharge diagnoses for participating states for each year. For certain states/years, the HCUP has linked each patient's data with subsequent inpatient or ED visits, thus allowing for longitudinal tracking of individual patients. Data are then deidentified and are available to researchers for a fee after completing a data use agreement. California was selected for this analysis as it is the most populous state and had linked data available from 2005 to 2011.

\section{Protocol Approval}

The study was approved by the University of California, San Francisco Human Research Committee, and the need for informed consent was waived due to the use of deidentified administrative data.

\section{Patients}

Adults $\geq 55$ years old were included in the cohort if they were diagnosed with TBI or NTT during an inpatient or ED visit in 2005 or 2006, did not die during the hospitalization, and did not have a diagnosis of PD or dementia in any discharge diagnosis field.

\section{Exposure}

TBI was defined using Centers for Disease Control and Prevention (CDC) criteria ${ }^{12,13}$ : International Classification of Diseases, Ninth Revision, Clinical Modification (ICD-9-CM) $800.0-801.9,803.0-804.9,850.0-854.1$, or 959.01 in any discharge diagnosis field. Mild TBI was defined according to CDC criteria $^{13}$ : ICD-9-CM first 4 digits 800.0, 800.5, 801.0, $801.5,803.0,803.5,804.0,804.5,850.0,850.1,850.5$, or 850.9 (with a fifth digit of $0,1,2,6,9$, or missing) or 854.0 (with a fifth digit of 1, 2, 6, 9, or missing). Moderate/severe TBI was defined as all nonmild TBI. NTT was defined as fracture, excluding fractures of the head and neck: ICD-9-CM $807.0-807.9,812-819.9,822-822.9$, or 823-827.9. Patients with both TBI and NTT during the same hospital visit were classified as TBI. We classified patients with multiple subsequent hospital visits based on their first visit only such that a patient who received a diagnosis of leg fracture during hospital visit 1 but received a diagnosis of TBI during hospital visit 2 was classified as NTT.

\section{Outcome}

The primary outcome was a diagnosis of PD (ICD-9-CM 332.0) made during a subsequent ED visit or inpatient hospitalization during the follow-up period ending in 2011. The follow-up in this study was comprised of all subsequent ED visits or inpatient hospitalizations that were recorded in the HCUP California SID or SEDD after the baseline visit for TBI or NTT. This allowed for a maximum follow-up of 5 to 7 years from the initial hospital visit for trauma. To further reduce the chance of reverse causation, patients were excluded if the diagnosis of PD was made $<1$ year after the trauma.

\section{Covariates}

Information was collected on age, sex, race/ethnicity, comorbidities (depression, ${ }^{14}$ delirium, ${ }^{15}$ drug/alcohol/tobacco disorders, and vascular risk factors including hypertension, hyperlipidemia, diabetes, coronary artery disease, peripheral vascular disease, and cerebrovascular disease), trauma mechanism, health care use, and trauma severity. ZIP Code-based median income quartile provided by the HCUP was included as a proxy for socioeconomic status. ${ }^{16}$ Comorbidities were based on ICD-9 discharge codes from the index visit for each patient as described previously ${ }^{8}$ with the addition of tobacco disorders/ dependence ICD-9-CM 305.1. Trauma mechanism was coded using major external cause of injury group codes $(\mathrm{E} \text { codes })^{17}$ and then divided into 4 categories: falls, vehicle accidents, assault, and other/unknown. An additional binary variable was generated denoting falls versus nonfalls. Health care use data included total hospital visits and total trauma visits per patient during the follow-up period including the index visit, as well as the location of the index visit (ED or inpatient). Trauma severity was defined according to the new injury severity score ${ }^{18}$ as described previously. ${ }^{8}$

\section{Primary Data Analysis}

All statistical analyses were performed using Stata 13.1. ${ }^{19}$ Summary statistics were generated for baseline characteristics and demographics of TBI and NTT groups and compared using $t$ test or chi-square test. Initial unadjusted estimates of risk of PD after TBI versus NTT were calculated using Kaplan-Meier estimates. Patients were not censored at death, as this information was not provided by the HCUP and deidentification precluded linkage to national death data. To evaluate the impact of potential confounders, we used Cox proportional hazard models adjusted for all covariates listed above (age category [defined as 
TABLE 1. Baseline Characteristics of Patients with TBI versus NTT

\begin{tabular}{|c|c|c|c|}
\hline Characteristics & NTT, $n=113,406$ & TBI, $\mathrm{n}=52,393$ & $p$ \\
\hline Age, yr & $70.9(10.9)$ & $73.4(11.1)$ & $<0.001$ \\
\hline $55-64$ & $40,355(35.6)$ & $14,653(28.0)$ & \\
\hline $65-74$ & $27,892(24.6)$ & $11,553(22.1)$ & \\
\hline $75-84$ & $29,265(25.8)$ & $15,784(30.1)$ & \\
\hline $85+$ & $15,894(14.0)$ & $10,403(19.9)$ & \\
\hline Women & $76,705(69.3)$ & $29,603(57.3)$ & $<0.001$ \\
\hline Race/ethnicity & & & $<0.001$ \\
\hline White & $75,797(66.8)$ & $34,558(66.0)$ & \\
\hline African American & $3,860(3.4)$ & $2,033(3.9)$ & \\
\hline Hispanic & $14,747(13.0)$ & 6,271 (12.0) & \\
\hline Asian & $4,163(3.7)$ & $3,318(6.3)$ & \\
\hline Other/missing & $14,839(13.1)$ & 6,213 (11.9) & \\
\hline Median income quartile & & & $<0.001$ \\
\hline 1st, poorest & $25,746(23.2)$ & $10,276(20.2)$ & \\
\hline 2nd & $26,856(24.2)$ & $12,184(23.9)$ & \\
\hline 3 rd & $29,811(26.9)$ & $14,327(28.1)$ & \\
\hline 4th, wealthiest & $28,436(25.6)$ & $14,132(27.8)$ & \\
\hline \multicolumn{4}{|l|}{ ICD-9 comorbidities at index visit } \\
\hline Hypertension & $34,820(30.7)$ & $18,139(34.6)$ & $<0.001$ \\
\hline Hyperlipidemia & $10,759(9.5)$ & $4,909(9.4)$ & 0.447 \\
\hline Diabetes & $15,398(13.6)$ & $7,193(13.7)$ & 0.404 \\
\hline Coronary artery disease & $8,971(7.9)$ & $5,143(9.8)$ & $<0.001$ \\
\hline Peripheral vascular disease & $1,319(1.2)$ & $581(1.1)$ & 0.335 \\
\hline Cerebrovascular disease & $2,416(2.1)$ & $2,007(3.8)$ & $<0.001$ \\
\hline Depression & $3,483(3.1)$ & $1,576(3.0)$ & 0.486 \\
\hline Delirium & $413(0.36)$ & $228(0.44)$ & 0.030 \\
\hline Drug disorder/dependence & $433(0.38)$ & $170(0.32)$ & 0.071 \\
\hline Alcohol disorder/dependence & $1,239(1.1)$ & $1,142(2.2)$ & $<0.001$ \\
\hline Tobacco use & $3,668(3.2)$ & $1,423(2.7)$ & $<0.001$ \\
\hline Trauma mechanism & & & $<0.001$ \\
\hline Fall & $75,352(66.4)$ & $34,831(66.5)$ & \\
\hline Vehicle accident & $9,886(8.7)$ & $7,448(14.2)$ & \\
\hline Assault & $827(0.7)$ & $1,585(3.0)$ & \\
\hline Other/missing & $27,341(24.1)$ & $8,529(16.3)$ & \\
\hline \multicolumn{4}{|l|}{ Health care use } \\
\hline Index visit location $=\mathrm{ED}$ & $77,128(68.0)$ & $35,767(68.3)$ & 0.298 \\
\hline Total inpatient or ED visits & $5.0(6.4)$ & $5.4(7.2)$ & $<0.001$ \\
\hline Total inpatient or ED visits for TBI/trauma & $1.33(0.7)$ & $1.31(0.7)$ & $<0.001$ \\
\hline
\end{tabular}


TABLE 1: Continued

\begin{tabular}{|c|c|c|c|}
\hline Characteristics & NTT, $n=113,406$ & TBI, $\mathrm{n}=52,393$ & $p$ \\
\hline New injury severity score & $5.0(3.7)$ & $7.8(5.9)$ & $<0.001$ \\
\hline TBI severity at index visit & & & $<0.001$ \\
\hline Mild TBI & N/A & $11,799(22.5)$ & \\
\hline Moderate/severe TBI & $\mathrm{N} / \mathrm{A}$ & $40,594(77.5)$ & \\
\hline TBI frequency & & & $<0.001$ \\
\hline 1 TBI anytime during study period & $5,950^{\mathrm{a}}(5.3)$ & $44,733(85.4)$ & \\
\hline$>1$ TBI anytime during study period & $1,101^{\mathrm{a}}(1.0)$ & $7,660(14.6)$ & \\
\hline \multicolumn{4}{|c|}{$\begin{array}{l}\text { Values are mean (standard deviation) or No. (\%). Total inpatient or ED visits are mean per participant over follow-up period } \\
\text { including index visit. } \\
\text { aThese patients were diagnosed with NTT at the index visit and then had subsequent ED or inpatient visit(s) for TBI. } \\
\text { ED = emergency department; ICD-9 = International Classification of Diseases, Ninth Revision; N/A = not applicable; } \\
\text { NTT = non-TBI trauma; TBI = traumatic brain injury. }\end{array}$} \\
\hline
\end{tabular}

55-64 years, 65-74 years, 75-84 years, or 85 years and older], sex, race/ethnicity, income, comorbidities, trauma mechanism, health care use, and new injury severity score). The time metameter for the Cox models was time since the index visit for TBI or NTT.

\section{Additional Analyses}

We tested for an interaction between TBI and trauma mechanism (falls, vehicle accidents, assault, and other/unknown) as well as between TBI and falls (falls vs nonfalls). We assessed the role of time lag from trauma to PD diagnosis by conducting separate analyses after excluding cases of PD diagnosed $<1$ year (primary analysis), 2 years, or 3 years after TBI or NTT. We assessed the roles of TBI severity and TBI frequency by using an expanded TBI variable in a single Cox model (NTT vs mild TBI vs moderate/severe TBI and NTT vs 1 TBI vs $>1 \mathrm{TBI}$ ). To test for a significant dose response for mild versus moderate/severe TBI and 1 versus $>1$ TBI (defined as a repeat TBI anytime during the study period), we used the Wald test. To test our hypothesis regarding age and TBI severity, we assessed for an interaction between age category and TBI severity as well as specifically between age category and mild $\mathrm{TBI}^{8}$ In a preplanned sensitivity analysis to account for loss to follow-up for any reason (including death), we excluded PD-free TBI and NTT patients whose last ED or inpatient visit recorded in the database was $>1$ year before the end of the follow-up period (defined as the period from the index visit until December 31, 2011). To account for potential misdiagnosis of secondary parkinsonism as PD or vice versa, we performed a final sensitivity analysis in which we excluded patients with a diagnosis of secondary parkinsonism (ICD-9-CM 332.1) at any time during the study period.

\section{Results}

\section{Primary Analysis}

A total of 165,799 cases of trauma were identified who did not have baseline PD or dementia and who did not die during the index hospitalization, and 52,393 (32\%) had TBI. Compared to the NTT patients, TBI patients were slightly older, were more likely to be male, were from higher income regions, had more comorbidities, and had higher injury severity scores (Table 1). Trauma was caused by falls in approximately $66 \%$ of both NTT and TBI patients. Median follow-up was 6 years (interquartile range $=5.5-6.5$ years). After exclusion of cases of PD that were diagnosed $<1$ year after TBI $(n=884)$, a total of 2,126 cases of PD were identified during the follow-up period. Patients with TBI were more likely to be diagnosed with PD compared to patients with NTT (1.7\% of TBI patients vs $1.1 \%$ of NTT patients, $p<0.001$; Fig 1). Patients with TBI were diagnosed with PD slightly sooner than those with NTT (average time to $\mathrm{PD}$ diagnosis $=3.1$ years vs 3.3 years, $\mathrm{p}=0.02$ ). Overall, patients diagnosed with PD had a mean age (at index visit) of 76 years (range $=55-95$, standard deviation $=8.6$ ), were $59 \%$ female, and were $68 \%$ white.

In the unadjusted model, TBI was associated with a $56 \%$ increased risk of PD diagnosis (Table 2). Individual adjustment for covariates changed the hazard ratio (HR) by $<10 \%$, except for age category. In the fully adjusted model (adjusted for age category, sex, race/ethnicity, income, comorbidities, trauma mechanism, health care use, and injury severity), TBI was associated with $44 \%$ increased risk of PD diagnosis (see Table 2). Results were similar if age was modeled as a continuous rather than a categorical variable.

\section{Additional Analyses}

In fully adjusted models, there was no interaction identified between trauma mechanism (defined as fall, vehicle accident, assault, or other/missing) and TBI status 


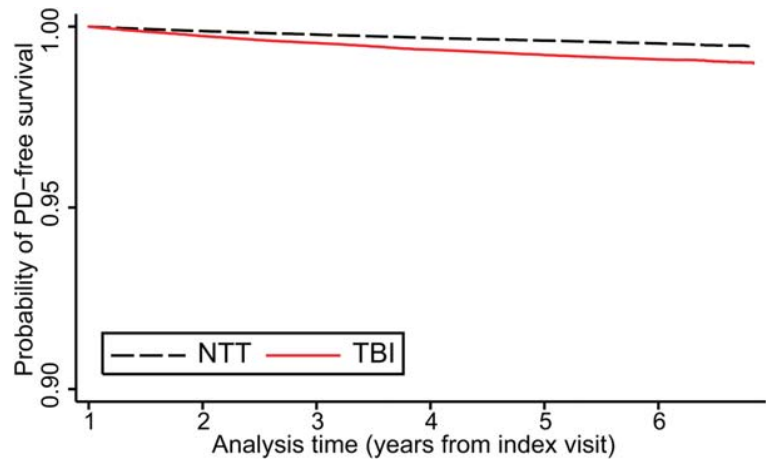

FIGURE 1: Kaplan-Meier plot showing Parkinson disease (PD)-free survival after traumatic brain injury (TBI) versus non-TBI trauma (NTT). TBI is associated with increased risk of PD compared to NTT. The Kaplan-Meier plot is adjusted for age. [Color figure can be viewed in the online issue, which is available at www.annalsofneurology.org.]

(interaction $p=0.21$ ) or between trauma mechanism and TBI severity (interaction $p=0.38$ ). Varying the time lag from trauma to PD diagnosis or including only trauma due to falls or only trauma due to nonfalls produced results essentially identical to the primary analysis (Fig 2). Furthermore, there was a significant dose response identified for TBI severity and TBI frequency such that risk of PD following more severe or more frequent TBI was doubled compared to that of mild or single TBI (see Fig 2). There was no interaction identified between age category and TBI severity (interaction $p=0.18$ ) or specifically between age category and mild TBI, after excluding moderate/severe TBI cases (interaction $p=0.77$ ). In a preplanned sensitivity analysis designed to account for loss to follow-up for any reason (including death) by excluding non-PD patients without a visit in the database within 1 year of the end of follow-up, results were similar to the primary analysis (fully adjusted $\mathrm{HR}=1.55$, $95 \%$ confidence interval $(\mathrm{CI})=1.41-1.70, p<0.001)$.

TABLE 2. Primary Analysis Cox Models Showing Risk of PD after TBI versus NTT

\begin{tabular}{lccl} 
& HR & $\mathbf{9 5 \%}$ CI & $\boldsymbol{p}$ \\
\hline Unadjusted & 1.55 & $1.43-1.70$ & $<0.001$ \\
\hline $\begin{array}{l}\text { Adjusted for } \\
\text { age-category only }\end{array}$ & 1.45 & $1.33-1.58$ & $<0.001$ \\
$\begin{array}{l}\text { Fully adjusted } \\
\text { for all covariates }\end{array}$ & 1.44 & $1.31-1.58$ & $<0.001$ \\
\hline
\end{tabular}

Fully adjusted model is adjusted for age, sex, race/ethnicity, income, comorbidities, trauma mechanism, health care use, and injury severity score.

$\mathrm{CI}=$ confidence interval; $\mathrm{HR}=$ hazard ratio; $\mathrm{NTT}=$ nonTBI trauma; $\mathrm{PD}=$ Parkinson disease; $\mathrm{TBI}=$ traumatic brain injury.

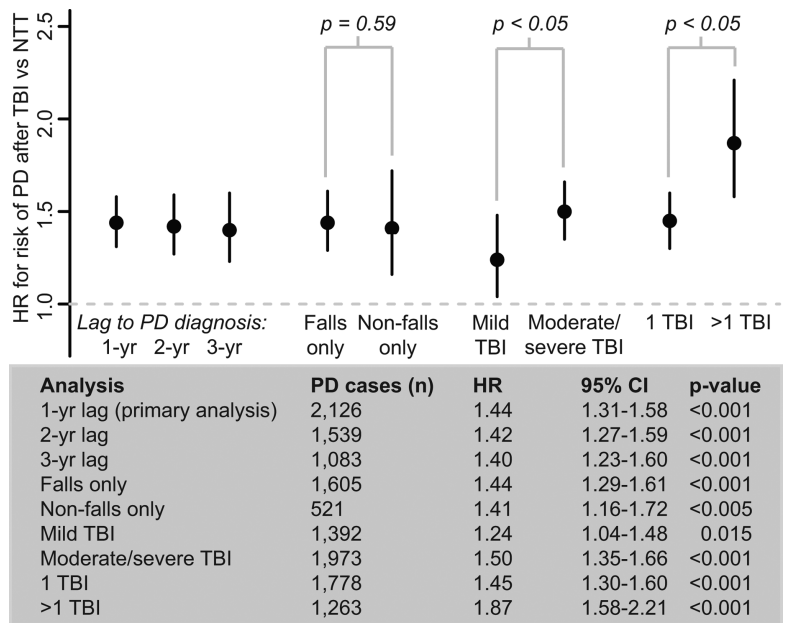

FIGURE 2: The role of time lag from trauma to Parkinson disease (PD) diagnosis, trauma mechanism (falls vs nonfalls), traumatic brain injury (TBI) severity, and TBI frequency. Excluding PD diagnosis rendered $<1$ year (primary analysis), $<2$ years, or $<3$ years after trauma led to essentially equivalent results. Analyzing only trauma due to falls versus only trauma due to nonfalls produced equivalent results ( $p$-value in figure is for interaction term for TBI $\times$ fall). Risk of PD after moderate/severe TBI was significantly greater than risk of PD after mild TBI ( $p$-value in figure is for Wald test). After excluding non-TBI trauma (NTT) cases who went on to suffer a TBI and then stratifying TBI cases by those with only $1 \mathrm{TBI}$ versus those who went on to suffer an additional TBI during the study period, risk of PD after $>1 \mathrm{TBI}$ was significantly greater than risk of PD after 1 TBI ( $p$-value in figure is for Wald test). Error bars are $95 \%$ confidence intervals $(\mathrm{Cls}) . \mathrm{HR}=$ hazard ratio.

Lastly, to account for potential misdiagnosis of secondary parkinsonism as PD or vice versa, after excluding all patients with a diagnosis of secondary parkinsonism at any time during the study period ( $\mathrm{n}=5$, of whom 1 also had a diagnosis of $\mathrm{PD}$ ), results were identical to the primary analysis (fully adjusted $\mathrm{HR}=1.44, \quad 95 \%$ $\mathrm{CI}=1.31-1.58)$.

\section{Discussion}

Among middle-aged and older patients diagnosed with trauma in an ED or inpatient setting, we found that there is a $44 \%$ increased risk of being diagnosed with PD over the subsequent 5 to 7 years after TBI compared to NTT. Furthermore, we found that risk is significantly higher with more severe or more frequent TBI, lending additional weight to a causal association.

This study is novel due to the use of NTT controls as a means to reduce possible confounding and reverse causation if patients with incipient PD are more likely to fall and sustain a TBI than healthy controls. The success of this approach is highlighted by our finding that approximately $66 \%$ of trauma was caused by falls in both the TBI and NTT groups. Furthermore, we found that risk of PD after TBI due to falls versus nonfalls is 
equivalent. This finding suggests that even if some patients who fall and sustain TBI are more likely to have incipient PD due to slower reaction times ${ }^{9}$ that may predispose to head rather than bodily injury, then the impact on the results is negligible. Additionally, the evidence for a dose response for increasing TBI severity and TBI frequency, and our persistently significant results despite multiple additional analyses, all enhance causal inference.

These results are in line with a recent meta-analysis of 22 studies that reported a pooled odds ratio of 1.57 for the association between PD and head trauma. ${ }^{4}$ In this meta-analysis, despite variability in methodological approach and statistical significance, nearly all (19 of 22) studies reported odds ratios $>1$. Aside from mounting evidence for an association between TBI and PD, many prior studies have identified TBI as an important risk factor for late onset dementia ${ }^{20-23}$ and possibly early onset dementia as well. ${ }^{24}$ Together, this body of work suggests that TBI may be an important risk-magnifier or threshold lowerer for neurodegeneration of many kinds.

The risk of PD following mild TBI in particular has been somewhat less clear. Results of the few prior studies on this topic have been mixed. For example, of the 5 qualifying studies analyzed in a systematic review of the literature from 1990-2012, ${ }^{5}$ only 2 reported an elevated risk of PD after mild TBI. ${ }^{6,25}$ Interestingly, the authors of one of these studies attributed these results to reverse causation $^{6}$; the authors of the other study, to suboptimal matching of controls. ${ }^{25}$ Our study appears to be among the largest to date to specifically assess the risk of PD following mild TBI while mitigating both of these prior methodological concerns. In our analysis of $>11,000$ patients with mild TBI compared to $>113,000$ patients with NTT, we identified $>1,300$ subsequent cases of PD. Patients with mild TBI were $24 \%$ more likely to develop PD than those with NTT. The lack of an interaction between age category and mild TBI indicates that risk of PD following mild TBI is similar across ages.

$\mathrm{PD}$ is a progressive neurodegenerative disorder characterized by loss of pigmented dopaminergic neurons of the substantia nigra as well as the presence of abnormal alpha-synuclein-containing Lewy bodies and Lewy neurites. ${ }^{26}$ Prior to development of clinically apparent parkinsonism, patients must lose upwards of $60 \%$ of striatal dopamine. ${ }^{27} \mathrm{~A}$ causal association between TBI and PD may be explained by several possible mechanisms. First, TBI may produce a static brain injury that reduces motor reserve, thereby leading to an earlier diagnosis of PD in a susceptible patient (eg, by unmasking otherwise subclinical symptoms). Second, TBI may actively accelerate or augment a pre-existing neurodege- nerative cascade. Third, TBI may trigger a de novo neurodegenerative cascade. Our results could theoretically lend support to the first 2 hypotheses, but the relatively short period of follow-up precludes commentary regarding the third hypothesis.

A number of prior studies using animal models of TBI support a causal mechanism for post-TBI PD. For example, a study of experimentally induced TBI in rats showed $15 \%$ loss of dopaminergic neurons ipsilateral to the injury just 11 days after injury that increased to $30 \%$ bilateral dopaminergic neuron loss 26 weeks postinjury. ${ }^{28}$ Others have shown persistently decreased markers of dopamine synthesis and abnormal accumulation of alphasynuclein in the substantia nigra 60 days after injury. ${ }^{29}$ Recently, studies in humans have begun to replicate some of these findings. Alpha-synuclein is elevated in cerebrospinal fluid of TBI patients compared to controls during the week following injury, and the degree of elevation is highly predictive of survival. ${ }^{30}$ Among patients who die after TBI, abundant alpha-synuclein deposition may be seen within injured axons. ${ }^{31}$ A preliminary autopsy analysis from the Adult Changes in Thought study that explored associations between an array of dementia-related neuropathologies and prior history of TBI among 525 patients (107 with TBI) found that alpha-synuclein was the only dementia-related neuropathology that was significantly associated with TBI history. ${ }^{32}$ Small studies in clinical populations have reported parkinsonism immediately following severe TBI that is sometimes dopamine responsive $^{33}$ and have identified functional magnetic resonance imaging abnormalities in motor networks among patients with post-traumatic parkinsonism that mirror those reported in idiopathic PD. ${ }^{34}$ Post-traumatic parkinsonism, however, may be transient and is hypothesized to be primarily due to traumatic axonal disruption of nigrostriatofrontal pathways. Among those cases that become chronic or progressive, it is conceivable that neurodegenerative pathology may be a contributing factor. This hypothesis, however, is currently speculative and requires further study. Lastly, some have found that TBI exposure may synergize with other environmental exposures, such as pesticides, ${ }^{2,28}$ or specific genes ${ }^{35,36}$ to increase risk for $\mathrm{PD}$, suggesting that certain subpopulations may be at particularly high risk for post-TBI PD.

This study is limited by the use of inpatient and ED administrative diagnostic codes, which may be poorly sensitive or specific to PD diagnoses. ${ }^{37,38}$ Poor sensitivity, if equal across groups, should not bias the relative magnitude of the association. However, severe TBI or bodily trauma may make a diagnosis of PD difficult due to the possibility of post-traumatic motor or behavioral abnormalities that may complicate assessment. Thus some degree of bias in 
diagnostic sensitivity across groups cannot be entirely ruled out. Given the constraints of this administrative data set, we were unable to validate PD diagnoses via expert review of medical records or to develop complex algorithms to include only diagnoses rendered by experts or to account for medication use. ${ }^{38}$ Thus, the possibility of misdiagnosis in this study underscores the critical importance of confirming these findings in large-scale prospective studies, ideally with autopsy confirmation. The study is additionally limited by lack of information regarding medical history (including prior TBI history) prior to the study period, lack of detailed information regarding acute management of TBI such as medications and surgical interventions, the relatively short 5- to 7-year follow-up duration, inability to censor at death or loss to follow-up for any reason, lack of outpatient data, and possible selection bias if patients who present to the hospital for TBI differ from those who do not seek medical care. ${ }^{39}$ Additionally, by using a trauma control group, we essentially controlled for any additional deleterious systemic effects of trauma on the nervous system that could potentially independently increase risk of PD. Thus, if NTT itself increases risk for PD, then the risk of PD following TBI may be underestimated in this study. Lastly, while the use of a NTT control group may reduce confounding, the possibility for residual confounding remains. Assault was a more common mechanism of injury and alcohol disorders/dependence were more common baseline comorbidities among TBI patients compared to NTT patients. Although we adjusted for these (and many other) potential confounders, we cannot exclude the possibility that some residual unmeasured confounders exist (eg, a behavior that may lead a person to be more likely to sustain a TBI vs an NTT and that may also be an independent risk factor for PD). Despite these limitations, we assert that the careful design of this study as well as the robustness of the multiple additional analyses and identification of a dose response support a causal association. We propose that future studies of neurodegenerative disease using this data set may be appropriate if either the outcome or predictor of interest is well suited to an inpatient or ED diagnosis (as in the case of incident TBI) and if the investigators carefully consider the above limitations.

\section{Conclusion}

We report that among middle-aged and older trauma patients presenting to an ED or inpatient setting, a TBI results in a $44 \%$ increased risk of PD compared to a trauma to the rest of the body over a follow-up period of just 5 to 7 years. Based on our careful study design and extensive secondary analyses, this result is almost certainly not solely due to reverse causation or confounding.
Furthermore, in combination with our prior study that identified a $26 \%$ increased risk of dementia after TBI versus NTT in this population, ${ }^{8}$ our results suggest that TBI is an important independent risk factor for a variety of neurodegenerative syndromes. Whether these post-TBI syndromes are primarily subserved by typical dementia or PD neuropathologies or may be partially or wholly due to unique TBI-specific neuropathology, such as has been documented in patients subjected to repeated TBI who have chronic traumatic encephalopathy, deserves further study. It is important to note that the vast majority of TBI patients in this study did not develop PD. This finding suggests that there must be multiple additional risk or protective factors that determine susceptibility or resilience to post-TBI neurodegeneration. Thus, it is imperative for future studies to continue to elucidate the underlying mechanisms and additional risk factors for post-TBI neurodegenerative disease to inform treatment and prevention in this high-risk population. Lastly, as the cause of trauma in this study was overwhelmingly due to falls, there is critical importance for fall prevention in middle-aged and older adults not only as a means to prevent bodily injury but potentially as a means to prevent neurodegenerative diseases such as dementia and PD.

\section{Acknowledgment}

This study was supported by the Department of Veterans Affairs Office of Academic Affiliations Advanced Fellowship Program in Mental Illness Research and Treatment (R.C.G.); Medical Research Service of the San Francisco Veterans Affairs Medical Center (R.C.G.); Department of Veterans Affairs Sierra-Pacific Mental Illness Research, Education, and Clinical Center (R.C.G.); the UCSF Pepper Center Research Career Development Core (R.C.G.); NIH (K24 AG031155, K.Y.; K08 NS082597, J.F.B.); Department of Defense (W81XWH-12-1-0581, K.Y.); Department of Veterans Affairs (K.Y.); California Department of Public Health (K.Y.); Bright Focus Foundation (K.Y.); and Alzheimer's Association (K.Y.). [Correction added on April 27, 2015, after first online publication: USCF support added for R.C.G.]. The authors thank the Healthcare Cost and Utilization Project for compiling and managing the data used in this study.

\section{Authorship}

R.C.G. contributed to the research project (conception, organization, and execution), statistical analysis (design and execution), and manuscript (writing). J.F.B. contributed to the research project (conception and organization), statistical analysis (design, review, and critique), 
and manuscript (review and critique). J.N. contributed to the research project (conception), statistical analysis (design, review, and critique), and manuscript (review and critique). S.G. contributed to the research project (conception), statistical analysis (design, review, and critique), and manuscript (review and critique). C.M.T. contributed to the research project (conception), statistical analysis (review and critique), and manuscript (review and critique). K.Y. contributed to the research project (conception), statistical analysis (review and critique), and manuscript (review and critique).

\section{Potential Conflicts of Interest}

K.Y.: grants, NIH—NIA, NIDDK, NIMH, Department of Defense, Veterans Administration, Bright Focus Foundation, Alzheimer's Association, California Department of Public Health; consultancy, Pfizer,Novartis; data and safety monitoring board, Takeda, NIA-sponsored study; board member, Beeson Scientific Advisory.

\section{References}

1. Faul M, Xu L, Wald MM, Coronado V, Dellinger AM. Traumatic brain injury in the United States: national estimates of prevalence and incidence, 2002-2006. Inj Prev 2010;16:A268-A.

2. Lee PC, Bordelon $Y$, Bronstein J, Ritz B. Traumatic brain injury, paraquat exposure, and their relationship to Parkinson disease. Neurology 2012;79:2061-2066

3. Goldman SM, Tanner CM, Oakes D, et al. Head injury and Parkinson's disease risk in twins. Ann Neurol 2006;60:65-72.

4. Jafari S, Etminan M, Aminzadeh F, Samii A. Head injury and risk of Parkinson disease: a systematic review and meta-analysis. Mov Disord 2013;28:1222-1229.

5. Marras C, Hincapie CA, Kristman VL, et al. Systematic review of the risk of Parkinson's disease after mild traumatic brain injury: results of the International Collaboration on Mild Traumatic Brain Injury Prognosis. Arch Phys Med Rehabil 2014;95(3 suppl):S238-S244.

6. Rugbjerg K, Ritz B, Korbo L, et al. Risk of Parkinson's disease after hospital contact for head injury: population based case-control study. BMJ 2008;337:a2494.

7. Coronado VG, Thomas KE, Sattin RW, Johnson RL. The CDC traumatic brain injury surveillance system: characteristics of persons aged 65 years and older hospitalized with a TBI. J Head Trauma Rehabil 2005;20:215-228.

8. Gardner RC, Burke JF, Nettiksimmons J, et al. Dementia risk after traumatic brain injury versus non brain trauma: the role of age and severity. JAMA Neurol 2014;71:1490-1497.

9. Johnson L, James I, Rodrigues J, et al. Clinical and posturographic correlates of falling in Parkinson's disease. Mov Disord 2013:28:1250-1256.

10. Agency for Healthcare Research and Quality, Healthcare Cost and Utilization Project. Overview of the state inpatient databases. 2013. Available at: http://www.hcup-us.ahrq.gov/sidoverview.jsp. Accessed March 16, 2015.

11. Agency for Healthcare Research and Quality, Healthcare Cost and Utilization Project. Overview of the state emergency department databases. 2013. Available at: http://www.hcup-us.ahrq.gov/seddoverview.jsp. Accessed March 16, 2015.
12. Thurman DJ, Sniezek JE, Johnson D, et al. Guidelines for surveillance of central nervous system injury. Atlanta, GA: Centers for Disease Control and Prevention, 1995.

13. National Center for Injury Prevention. Report to Congress. Mild traumatic brain injury in the United States: steps to prevent a serious public health problem. Atlanta, GA: Centers for Disease Control and Prevention, 2003.

14. Byers AL, Covinsky KE, Barnes DE, Yaffe K. Dysthymia and depression increase risk of dementia and mortality among older veterans. Am J Geriatr Psychiatry 2012;20:664-672.

15. Davis $\mathrm{DH}$, Muniz Terrera $\mathrm{G}$, Keage $\mathrm{H}$, et al. Delirium is a strong risk factor for dementia in the oldest-old: a population-based cohort study. Brain 2012;135(pt 9):2809-2816.

16. Yaffe K, Falvey C, Harris TB, et al. Effect of socioeconomic disparities on incidence of dementia among biracial older adults: prospective study. BMJ 2013;347:f7051

17. LeMier M, Cummings $P$, West TA. Accuracy of external cause of injury codes reported in Washington State hospital discharge records. Inj Prev 2001;7:334-338.

18. Osler T, Baker SP, Long W. A modification of the injury severity score that both improves accuracy and simplifies scoring. J Trauma 1997;43:922-925; discussion 925-926.

19. StataCorp. Stata Statistical Software: release 13. College Station, TX: StataCorp, 2013

20. Mortimer JA, van Duijn CM, Chandra V, et al. Head trauma as a risk factor for Alzheimer's disease: a collaborative re-analysis of case-control studies. EURODEM Risk Factors Research Group. Int J Epidemiol 1991;20(suppl 2):S28-S35

21. Plassman BL, Havlik RJ, Steffens DC, et al. Documented head injury in early adulthood and risk of Alzheimer's disease and other dementias. Neurology 2000;55:1158-1166.

22. Barnes DE, Kaup A, Kirby KA, et al. Traumatic brain injury and risk of dementia in older veterans. Neurology 2014;83:312-319.

23. Fleminger $\mathrm{S}$, Oliver $\mathrm{DL}$, Lovestone $\mathrm{S}$, et al. Head injury as a risk factor for Alzheimer's disease: the evidence 10 years on; a partial replication. J Neurol Neurosurg Psychiatry 2003;74:857-862

24. Nordstrom P, Michaelsson K, Gustafson Y, Nordstrom A. Traumatic brain injury and young onset dementia: a nationwide cohort study. Ann Neurol 2014;75:374-381.

25. Seidler A, Hellenbrand W, Robra BP, et al. Possible environmental, occupational, and other etiologic factors for Parkinson's disease: a case-control study in Germany. Neurology 1996;46:12751284.

26. Spillantini MG, Crowther RA, Jakes R, et al. Alpha-synuclein in filamentous inclusions of Lewy bodies from Parkinson's disease and dementia with Lewy bodies. Proc Natl Acad Sci U S A 1998;95: 6469-6473.

27. Bernheimer H, Birkmayer W, Hornykiewicz O, et al. Brain dopamine and the syndromes of Parkinson and Huntington. Clinical, morphological and neurochemical correlations. J Neurol Sci 1973; 20:415-455

28. Hutson CB, Lazo CR, Mortazavi F, et al. Traumatic brain injury in adult rats causes progressive nigrostriatal dopaminergic cell loss and enhanced vulnerability to the pesticide paraquat. J Neurotrauma 2011;28:1783-1801.

29. Acosta SA, Tajiri N, de la Pena I, et al. Alpha-synuclein as a Pathological Link between Chronic Traumatic Brain Injury and Parkinson's disease. J Cell Physiol 2015;230:1024-1032.

30. Mondello S, Buki A, Italiano D, Jeromin A. Alpha-synuclein in CSF of patients with severe traumatic brain injury. Neurology 2013;80: 1662-1668.

31. Uryu K, Chen XH, Martinez D, et al. Multiple proteins implicated in neurodegenerative diseases accumulate in axons after brain trauma in humans. Exp Neurol 2007;208:185-192. 
32. Keene CD, Gibbons LE, Sonnen JA, et al. Associations between lifetime history of traumatic brain injury and dementia-related neuropathological findings at autopsy: the Adult Changes in Thought study. Paper presented at: Alzheimer's Association International Conference; July 2014; Copenhagen, Denmark.

33. Formisano R, Zasler ND. Posttraumatic parkinsonism. J Head Trauma Rehabil 2014;29:387-390.

34. Peran P, Catani S, Falletta Caravasso C, et al. Supplementary motor area activation is impaired in severe traumatic brain injury parkinsonism. J Neurotrauma 2014;31:642-648.

35. Goldman SM, Kamel F, Ross GW, et al. Head injury, alpha-synuclein Rep1, and Parkinson's disease. Ann Neurol 2012;71:40-48.
36. Lee PC, Bordelon $Y$, Bronstein J, et al. Head injury, $\alpha$-synuclein genetic variability and Parkinson's disease. Eur J Neurol 2014 Nov 5.

37. Feldman AL, Johansson AL, Gatz M, et al. Accuracy and sensitivity of Parkinsonian disorder diagnoses in two Swedish national health registers. Neuroepidemiology 2012;38:186-193.

38. Szumski NR, Cheng EM. Optimizing algorithms to identify Parkinson's disease cases within an administrative database. Mov Disord 2009;:24:51-56.

39. Setnik L, Bazarian JJ. The characteristics of patients who do not seek medical treatment for traumatic brain injury. Brain Inj 2007 21:1-9. 